\title{
An Efficient Low Level Features of CBIR using Wavelet, GLDM and SMOSVM Method
}

\author{
Dharmendra Pandey $^{1}$ and Shivpratap kushwah ${ }^{2}$ \\ Dept. of CSE/IT \\ ITM Universe, Gwalior, India \\ Dharmendra.pandey81@gmail.com ${ }^{1}$ and shivpratapkushwah@gmail.com²
}

\begin{abstract}
Content Based Image Retrieval (CBIR) is a process that allows for a person to extract an image centered on a question, from a database containing an enormous amount of pictures. A very fundamental issue in designing a CBIR system is to select the image features that best represent the image contents in a database. In this research, efficient low level features of CBIR using Bi-cubic interpolation (BCI) with color coding (CC), gray level difference method (GLDM), Hu moments and four levels of discrete wavelet transform (DWT). In this paper the techniques of CBIR are discussed, analyzed and compared. BCI is used to scale the query image and database images. CC is used for color feature extraction. Apply DWT and GLDM on each level plane of an image for texture feature. Apply $\mathrm{Hu}$ moments are used for shape features. The experimental database performed on Corel database which contain fruit, flowers, sports, tools, facial images. Apply Sequential Minimal Optimization Support vector matching (SMOSVM) for classifying the data. The performance analysis of precision $(P)$, execution time $(T)$ for retrieving images. We calculate similarity distance on Euclidean distance (ED), Manhattan Distance (MD), City Block (CBD) and Canberra distance (CD).
\end{abstract}

Keywords: GLDM, SVM, DWT, CBIR, Hu moment, BCI, Precision, Recall.

\section{Introduction}

The rapid growth of special types of digital images has made it necessary to develop systems that organize and index the images for easy access. Physical annotation or text founded indexing is one method of doing this. Nonetheless, due to precise risks of textfounded indexing the additional manners of arranging and indexing of pictures like CBIR has been proposed. Original CBIR programs perform retrieval on the groundwork of features reward within the picture reminiscent of color, texture, or shape. The characteristic vectors of the query picture and database graphics are in comparison with every other to retrieve visually an identical picture [1]. This method contains no manual annotation and retrieves visually similar images. Hence CBIR is the necessitate of the hour [2].

Color, being a great descriptor, has been exploited a set of feature for image retrieval. Color based technique use tools such as histogram [3], and color correlogram [4] for indexing. Texture is an additional feature which has been in use. Texture represents structural association of neighborhood and describes smoothness, roughness, coarseness of the region [5]. Shape is also a normally used feature for retrieval [6]. Image retrieval using shape feature generally requires segmentation of objects for shape retrieval [7]. Lack of good segmentation algorithm makes this task a little difficult.

\section{GLDM}

GLDM is used for feature extraction [8] which estimates the likelihood Density capabilities for a given picture. For a given, image power function $\mathrm{I}(\mathrm{x}, \mathrm{y})$ and displacement $\delta=(\Delta \mathrm{x}, \Delta \mathrm{y})$, the value of probability density function $\mathrm{f}(\mathrm{i} \mid \delta)$ is estimated 
from the number of times modify in strength $I \delta(x, y)$, take place for a given displacement $d$, i.e $f(i \mid \delta)=P(I \delta(x, y)=i)$. In this four possible forms of inter sample spacing distance $d$ were considered: $(0, d),(d, 0),(-d, d)$, and $(-d,-d)$. Other than GLDM system, primary second and Zernike moments [9] are additionally obtained for scale, translation and rotation normalization respectively.

\section{Discrete Wavelet Transforms (DWT)}

The Wavelet transform is created via repeatedly filtering the picture coefficients on a row by way of row and column by column foundation. A two-dimensional DWT decomposition of the picture comprises quite a grouping of band information reminiscent of low-low frequency approximation band, high-low frequency vertical element band, low-high frequency horizontal detail band and excessive-high frequency diagonal element band. DWT is used to obtain just right picture retrieval base on the low computational fee. DWT, which transforms a discrete time sign to a discrete wavelet representation [10].

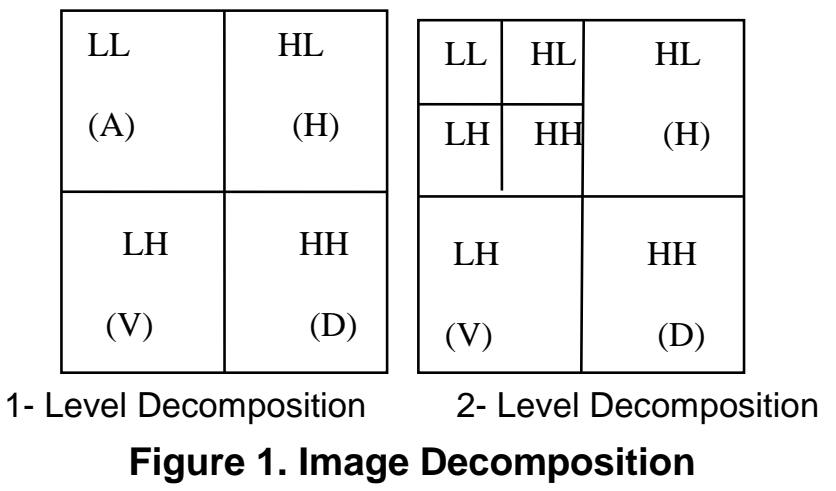

Wavelet Feature Extraction

Wavelet $\psi(\mathrm{t})$ is a function that satisfy the following condition

$\psi_{a, b}(t)=\frac{1}{\sqrt{a}} \psi\left(\frac{t-b}{a}\right)$

DWT is a spectral estimation technique, which decompose a function or a signal into extraordinary frequency sub-bands and leads to a set of wavelet coefficients. The low level physical features of the original image are buried in these wavelet coefficients; therefore the signal can be characterized by this wavelet coefficient [10].

\section{HU Moment}

Hu-Moment [11] in 1962 proposed seven properties related to connected region that are invariant to rotation, scaling, and translation (RTS) and are also known as Algebraic moment Invariants. 2Dimention moments of order $(p+q)$ for digital picture $f(x, y)$ is outlined as follows

$m_{p q}=\sum_{x} \sum_{y} x^{p} y^{q} f(x, y)$

Where, $\mathrm{p}, \mathrm{q}=0,1,2 \ldots$.

The summations are over the principles of spatial co-ordinates $x, y$ on both sides of the full picture. The moments in Equation. (2) are not in general invariant under translation, rotation or scale changes in the picture $\mathrm{f}(\mathrm{x}, \mathrm{y})$. Translation invariance may also be completed with the aid of utilizing crucial moment outlined as follows.

$$
\begin{array}{r}
\mu_{p q}=\sum \sum(x-\bar{x})^{p}(y-\bar{y})^{q} f(x, y) \\
\text { where, } \bar{x}=\frac{m_{10}}{m_{\infty}} \text { and } \bar{y}=\frac{m_{10}}{m_{\infty}}
\end{array}
$$

The normalized valuable moment of order $(p+q)$ is distinct. 


\section{Support Vector Machine}

Vapnik proposed SVM in 1979 (Vapnik, 1995)[12], However they have got handiest just lately been gaining status within the finding out community. The most important plan of an SVM is to construct a hyper plane as the decision floor in one of these means that the margin of separation among constructive and terrible examples is maximized. The thought which is middle to the construction of the support vector learning algorithm is the inner product kernel among a support vector $\mathrm{Xi}$ and a vector $\mathrm{X}$ drawn from the input space. The support vectors represent a little set of the tutorial info extracted by the support vector looking for algorithm program. The separation among the hyper plane and closet information is named the margin of separation, denoted by $\rho$.

The goal of a SVM is to find a particular hyper plane for which the margin of separation $\rho$ is maximized. Below this condition, the choice surface is spoken because the best hyper plane. The support vectors play a distinguished role within the operation of this category of learning machines. In abstract terms, the support vectors are those knowledge points that lie nearest to the choice surface, and thus the foremost troublesome to classify. They need a right away touching on the optimum location of the choice surface. The concept of an SVM relies on the subsequent 2 mathematical operations:

1. Nonlinear mapping of an enter pattern vector on to an upper dimensional function space that's secret from each the input and output.

2. Construction of associated degree best plane for separating the patterns within the higher dimensional aria obtained from operation1.

\section{Literature Survey}

[12] CBIR uses the visible contents of a picture like global features-color feature, shape feature, texture feature, and nearby features-spatial domain reward to propose and index the picture. On this paper worked on Haar DWT for decaying a picture into vertical and diagonal neighborhood band horizontal and GLCM for function take away. In this paper for categorization development, SVM used. The experimental results show improved results in comparison to previous methods.

[2] Shrink proposes an image retrieval system founded on moments of wavelet turn into. DWT coefficients of grayscale picture are computed which can be then normalized making use of z-score normalization Geometric moments of these normalized coefficients are computed to construct feature vectors. These characteristic vectors are used to retrieve visually identical pictures from large database. Performance of the proposed approach is validated on Corel-a thousand database and measured using precision d keep in mind parameters. The experimental outcomes show that the projected technique outperforms a number of the opposite state-of-the art strategies in terms of preciseness and recall.

[13] This paper a technique used to insert a watermark into scanned colored PDF files. The watermarking goals to protect the copyright possession and attest the PDF documents. An emblem has been used as a strong watermark for copyright. The logo will be embedded in the green channel of the file. This watermark can be successfully extracted and it can survive against different attacks. Any manipulations in the PDF file will cause the second fragile watermark to change and indicate forgery. The algorithm was successfully tested on different colored PDF files.

[14] SVM and color string coding feature selection. Overall our contribution, within the ever growing area of image databases is an efficient process to obtain images from huge database. Using database classification we can get better the performance of the CBIR than compared with normal CBIR i.e. without database classification. Finally, this database classification and color string coding feature selection give the better results.

[15] Proposed a wavelet based image retrieval by using signatures constructed from wavelet coefficients. This method has been tested on medical database and consumes a lot of time to retrieve results.

[16] Proposed another approach for CBIR by analyzing different families of wavelets and similarity metrics. This method works for those images in which objects are well 
defined and separated. Also, presence of error during reconstruction using wavelet transform and the presence of complex background in the image makes it unsuitable for certain types of images.

\section{Proposed Algorithm}

In this research, we improved the problem of retrieving time of the algorithm and accurate results. First of all, split the image into three planes: Red (R), Green $(G)$ and Blue (B). Then apply BCI on query image and database images. Apply CC, resize all frames to reduce the property of variation in size. Considering that the frames will have exceptional sizes, all frames are normalized to a general dimension (i.i. $20 \times 20$ pixels) in this step.in this, every one frames are resized by using the BCI process. After the switch, each frame will emerge as a $2 \mathrm{~d}$ string array, after which we will be able to convert the 2 nd string array to a string as below:

CCCCCCCCCCCCHCBCCCCC...RRRRRRRRRRRRRRRRRRRR $(20 \times 20=400$ characters) We will perceive the discrimination power among quite a lot of frames due to the fact 400 characters here 6400 diversifications. Perform 4-DWT on Red plane, Green plane and Blue plane.

1. Consider RGB image and remove the Red, Green, and Blue Components from an image.

2. Resize the image with $20 * 20$ sizes and apply cubic interpolation on the image.

3. We have an understanding of that the values of $r, g$, or b are entirely exclusive with the changed illumination stipulations. However, the relative values between $\mathrm{r}$ (i), g (i), and b (i) are very similar. Therefore, utilize 6 rules to move each frame to a color string as follow:

(1) If a pixel $\mathrm{R}>\mathrm{G}>\mathrm{B}$, then allots the pixel as ' $\mathrm{R}$ ';

(2) If a pixel $R>B>G$, then provides the pixel as $K(i, j, 1)=$ 'S';

(3) If a pixel $G>R>B$, then allots the pixel as $K(i, j, 2)=$ ' $G$ ';

(4) If a pixel $G>=B>=R$, then gives the pixel as $K(i, j, 2)=$ ' $H$ ';

(5) If a pixel $\mathrm{B}>=\mathrm{R}>=\mathrm{G}$, then assign the pixel as $\mathrm{K}(\mathrm{i}, \mathrm{j}, 3)=\mathrm{B}$ ';

(6) If a pixel $B>=G>=R$, then provide the pixel as $K(i, j, 3)=$ ' $C$ ';

1. Extract features of an image using gray level dissimilar method (entropy, mean, energy, homogeneity and contrast).

$$
\text { hom }=\sum \frac{P}{\text { greydiff } f^{2}+1}
$$

Where greydiff $=[0: 1], \mathrm{P}$ is probability function and hom denotes to homogeneity

con $=\sum\left(P X\right.$ greydiff $\left.^{2}+1\right)$

Where con is contrast of an image

eng $=\sum(P)^{2}$

Where eng denotes energy of an image

ent $=-\sum P X \log (P+$ eps $)$

Where ent denotes the entropy of an image

$B 1=\sum$ PXgreydiff

Where $\mathrm{B} 1$ is mean of an image

4. Apply 4-DWT to get estimated coefficient and vertical, horizontal and diagonal detail coefficients for red, green and blue components.

$[F R, F G, F B]=$ fourDWT(image)

Where FR, FG and FB are a feature vector of four level coefficient of DWT, image is query image.

5. Calculate moment $(p, q)$ of an image $f(x, y)$ of size $M^{*} N$ is defined using this equation:

$$
m_{p, q}=\sum_{x=0}^{M-1} \sum_{y=0}^{N-1} f(x, y) x^{p} y^{q}
$$

Where $p$ is the order of $x$ and $q$ is the order of $y$ 
6. A central moment is basically the equal as the moments just described besides that the values of $\mathrm{x}$ and $\mathrm{y}$ used within the formulation are displaced by the mean values

$$
\beta_{p, q}=\sum_{x=0}^{M-1} \sum_{y=0}^{N-1} f(x, y)\left(x-x_{\text {avg }}\right)^{p}\left(y-y_{a v g}\right)^{q}
$$

Where $x_{\text {avg }}=\frac{m_{10}}{m_{00}}$ and $y_{\text {avg }}=\frac{m_{01}}{m_{00}}$

7. The normalized moments $\gamma_{p, q}$ are the equal as the central moments except that they are all separated by an suitable power of $\mathrm{m}_{00}$

$\gamma_{p, q}=\frac{\gamma_{p, q}}{m_{00} \frac{p+q}{2}+1}$

8. Hu invariant moments are linear combinations of the central moments and here is how are defined eight $\mathrm{Hu}$ moments:

$$
\begin{gathered}
I 1=\gamma_{20}+\gamma_{02} \\
I 2=\left(\gamma_{20}-\gamma_{02}\right)^{\wedge}+4 \times \gamma_{11} \\
I 3=\left(\gamma_{30}-3 \times \gamma_{12}\right)^{\wedge} 2+\left(\gamma_{03}-3 \times \gamma_{21}\right)^{\wedge} 2 \\
I 4=\left(\gamma_{30}+\gamma_{12}\right)^{\wedge} 2+\left(\gamma_{03}+\gamma_{21}\right)^{\wedge} 2 \\
I 5=\left(\gamma_{30}-3 \times \gamma_{12}\right) \times\left(\gamma_{30}+\gamma_{12}\right) \times\left(\left(\gamma_{30}+\gamma_{12}\right)^{\wedge} 2-3 \times\left(\gamma_{21}+\gamma_{03}\right)^{\wedge} 2\right) \\
+\left(3 \times \gamma_{21}-\gamma_{03}\right) \times\left(\gamma_{21}+\gamma_{03}\right) \times\left(3 \times\left(\gamma_{30}+\gamma_{12}\right)^{\wedge} 2-\left(\gamma_{30}\right.\right. \\
\left.\left.+\gamma_{12}\right)^{\wedge} 2\right) \\
I 6=\left(\gamma_{20}-\gamma_{02}\right) \times\left(\left(\gamma_{30}+\gamma_{12}\right)^{\wedge} 2-\left(\gamma_{21}+\gamma_{03}\right)^{\wedge} 2\right)+4 \times\left(\gamma_{30}+\gamma_{12}\right) \times\left(\gamma_{21}\right. \\
\left.+\gamma_{03}\right) \\
I 7=\left(3 \times \gamma_{21}-\gamma_{03}\right) \times\left(\gamma_{30}+\gamma_{12}\right) \times\left(\left(\gamma_{30}+\gamma_{12}\right)^{\wedge} 2-3 \times\left(\gamma_{21}+\gamma_{03}\right)^{\wedge} 2\right) \\
+\left(\gamma_{03}-3 \times \gamma_{21}\right) \times\left(\gamma_{21}+\gamma_{03}\right) \times\left(3 \times\left(\gamma_{30}+\gamma_{12}\right)^{\wedge}-\left(\gamma_{30}\right.\right. \\
\left.\left.+\gamma_{12}\right)^{\wedge} 2\right) \\
I 8=\left(\gamma_{11} \times\left(\gamma_{30}+\gamma_{12}\right)^{\wedge} 2-\left(\gamma_{30}+\gamma_{12}\right)^{\wedge} 2-\left(\gamma_{20}-\gamma_{02}\right) \times\left(\gamma_{30}+\gamma_{12}\right)\right. \\
\times\left(\gamma_{21}+\gamma_{03}\right)
\end{gathered}
$$

9. do again step1 to step8 on a query picture in the database.

10. Determine the similarity matrix of query image and image database using Euclidean distance, Canberra, Manhattan and City block distance.

11. We have used Euclidean Distance, $E D$ using below equation. Methods used to arrange images, moreover, compute the distinction or comparison between two vectors. Given two vectors $Q$ and $D$, where

$$
\begin{array}{r}
Q=\left[\begin{array}{c}
q_{1} \\
q_{2} \\
\vdots \\
q_{n}
\end{array}\right] \text { and }=\left[\begin{array}{c}
d_{1} \\
d_{2} \\
\vdots \\
d_{n}
\end{array}\right] \\
\mathrm{d}(\mathrm{Q}, \mathrm{D})=\sum_{\mathrm{m}} \sum_{\mathrm{n}} \mathrm{d}_{\mathrm{mn}}(\mathrm{Q}, \mathrm{D}) \\
\text { Whered }_{\mathrm{mn}}=\frac{\left|\left(\mu_{\mathrm{mn}}^{\mathrm{Q}}-\mu_{\mathrm{mn}}^{\mathrm{D}}\right)\right|}{\left|\mu_{\mathrm{mn}}^{\mathrm{Q}}\right|+\left|\mu_{\mathrm{mn}}^{\mathrm{D}}\right|}+\frac{\left|\left(\mu_{\mathrm{mn}}^{\mathrm{Q}}-\mu_{\mathrm{mn}}^{\mathrm{D}}\right)\right|}{\left|\mu_{\mathrm{mn}}^{\mathrm{Q}}\right|+\left|\mu_{\mathrm{mn}}^{\mathrm{D}}\right|}
\end{array}
$$

12. Repeat the steps from 1 to 8 for all the images in the database.

13. Classify the images using SMOSVM classifier and combine low level features.

14. Calculate accuracy, precision, and recall of retrieved images.

$$
\begin{aligned}
& P=\frac{\text { No. ofrelevantimageretrieved }}{\text { Totalnumberofimageretrieved }} \\
& R=\frac{\text { No. ofrelevantimageretrieved }}{\text { numberofimageinthedatebese }}
\end{aligned}
$$




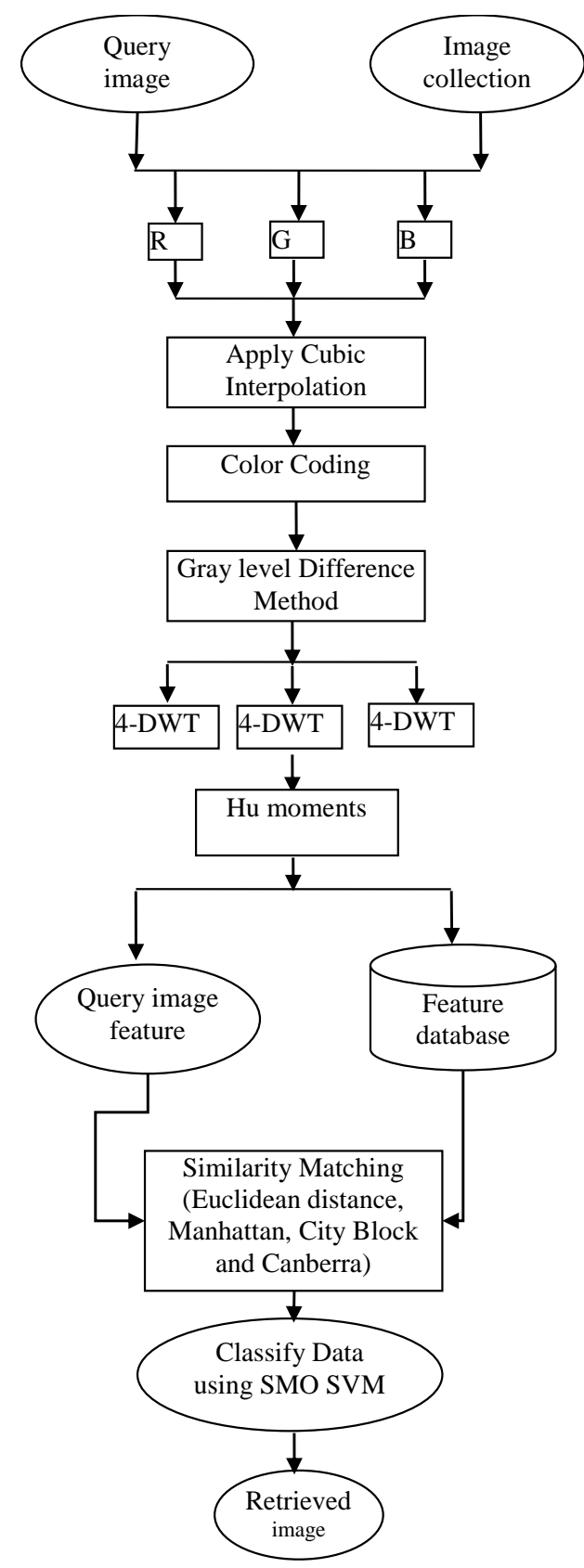

Figure 2. Block Diagram of Proposed Work

\section{Result Analysis}

In this work, the proposed method is performed by conducting experiments on Corel1085 database. This database, including sports, flowers, fruits, tools and leaf images. This database, including sports, flowers, fruits, tools and daintree images. First four categories contain 250 images and daintree image contain 85 with size of $20 * 20$. The experimental checked by different number of returning images which varies from 10 to 50. Calculate distance using $\mathrm{ED}, \mathrm{MD}, \mathrm{CBD}$ and $\mathrm{CD}$ give better result as compared to $\mathrm{ED}$.

A. Euclidean Distance:

In the process of a query image compute the distance between the transformed feature vector of each image of the query image and that of 1000 images in the database this distance is measured as: 


$$
\operatorname{dist}(p, d)=\frac{1}{10} \sqrt{\sum_{k=1}^{10}\left[\operatorname{dist}\left(q_{k}, d_{k}\right)\right]^{2}}
$$

Where $\mathrm{q}$ is query image and $\mathrm{d}$ is distance.

City blocks distance:

In this we are getting the value which is greater than or equal to $0 . I f 0$ comes then identical points are considered, and when it is greater than 0 there it is low similarity.

$\operatorname{dist}(q, d)=\frac{1}{10} \sum_{k=1}^{10}\left[\left[\operatorname{dist}\left(q_{k}, d_{k}\right)\right]^{2}\right]$

Precision-It is outlined because the quantitative relation of variety the quantity of retrieved relevant pictures to the entire number of retrieved pictures. We have a propensity to indicate the preciseness by $P$ [3]

$p=\frac{\text { Number of relevant images are retrived }}{\text { Total number of images retrived }}$

Recall-it's outlined because the magnitude relation of range the quantity of retrieved relevant pictures to the entire number of relevant pictures within the info. We denote to the recall by $R$ [3].

$R=\frac{\text { Number of relevant images are retrived }}{\text { Total number of relevant images in database }}$
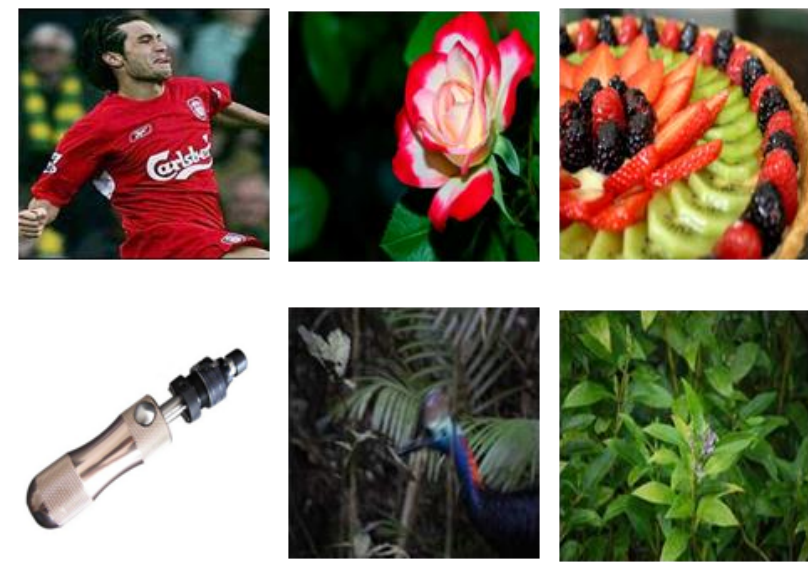

Figure 3. Corel -1085 Dataset

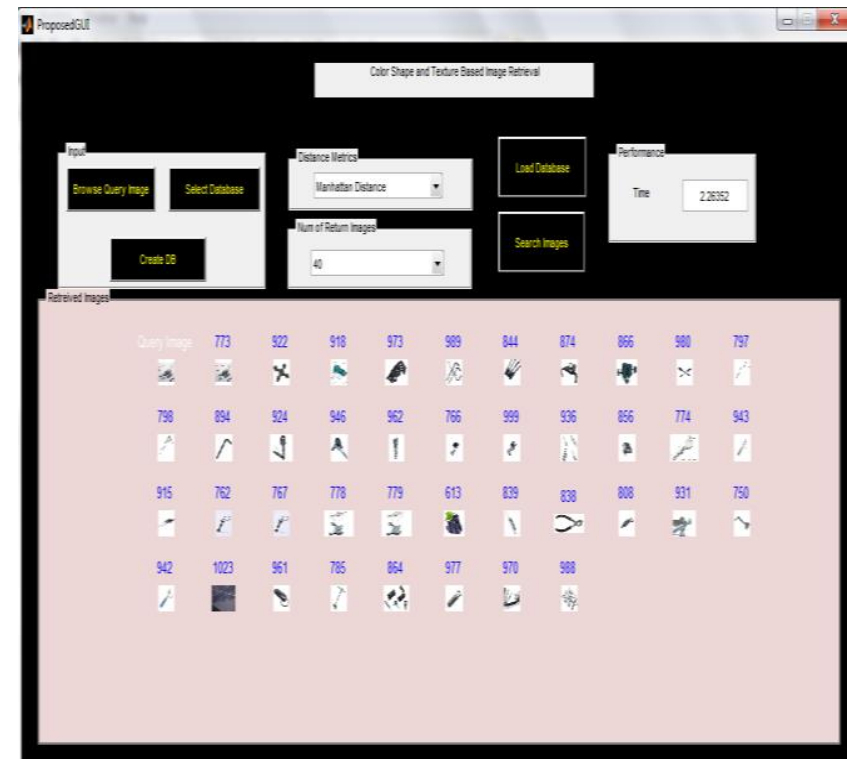

Figure 4. Results Shows on Tool Category 


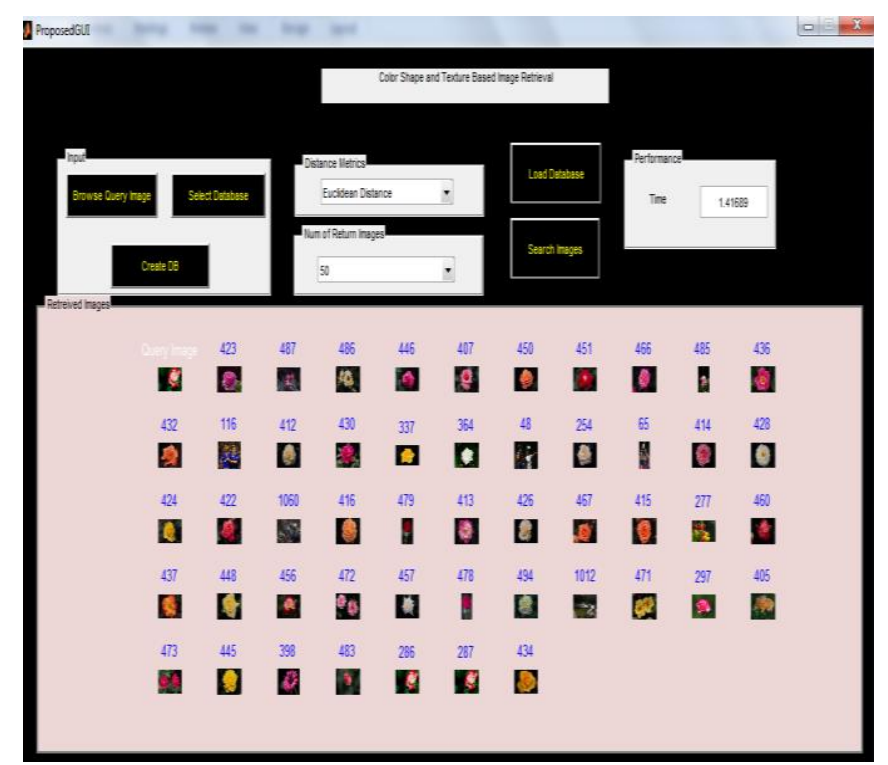

Figure 5. Results Shows on Flower Category

Table 1. Proposed precision tools image

\begin{tabular}{|l|l|l|l|l|l|}
\hline \multirow{2}{*}{$\begin{array}{l}\text { Similarity } \\
\text { Measure }\end{array}$} & \multicolumn{5}{|l|}{ Proposed Precision (\%) } \\
\cline { 2 - 6 } & $\mathbf{1 0}$ & $\mathbf{2 0}$ & $\mathbf{3 0}$ & $\mathbf{4 0}$ & $\mathbf{5 0}$ \\
\hline ED & 83.33 & 86.41 & 82.91 & 84.69 & 85.05 \\
\hline CBD & 78.95 & 76.79 & 80.73 & 81.13 & 85.19 \\
\hline CD & 75.00 & 79.53 & 85.12 & 88.89 & 86.00 \\
\hline MD & 82.24 & 84.30 & 80.00 & 84.11 & 76.79 \\
\hline
\end{tabular}

Table 2. Base Precision for Tools Image

\begin{tabular}{|l|l|l|l|l|l|}
\hline Similarity & \multicolumn{4}{|l|}{ Base Precision (\%) } \\
\cline { 2 - 6 } Measure & $\mathbf{1 0}$ & $\mathbf{2 0}$ & $\mathbf{3 0}$ & $\mathbf{4 0}$ & $\mathbf{5 0}$ \\
\hline ED & 82.86 & 82.22 & 82.01 & 82.95 & 83.82 \\
\hline
\end{tabular}

Table 3. Proposed Precision for Flower Image

\begin{tabular}{|l|l|l|l|l|l|}
\hline \multirow{2}{*}{$\begin{array}{l}\text { Similarity } \\
\text { Measure }\end{array}$} & \multicolumn{5}{|l|}{ Proposed Precision (\%) } \\
\cline { 2 - 6 } & $\mathbf{1 0}$ & $\mathbf{2 0}$ & $\mathbf{3 0}$ & $\mathbf{4 0}$ & $\mathbf{5 0}$ \\
\hline ED & 82.73 & 84.40 & 80.87 & 84.11 & 82.83 \\
\hline CBD & 83.33 & 83.33 & 81.48 & 81.08 & 82.03 \\
\hline CD & 78.50 & 81.90 & 83.49 & 83.33 & 84.16 \\
\hline MD & 83.50 & 81.98 & 82.30 & 86.73 & 80.00 \\
\hline
\end{tabular}

Table 4. Base Precision for Flower Image

\begin{tabular}{|l|l|l|l|l|l|}
\hline Similarity & \multicolumn{5}{|l|}{ Base Precision (\%) } \\
\cline { 2 - 6 } Measure & $\mathbf{1 0}$ & $\mathbf{2 0}$ & $\mathbf{3 0}$ & $\mathbf{4 0}$ & $\mathbf{5 0}$ \\
\hline ED & 82.01 & 80.13 & 79.86 & 82.39 & 81.51 \\
\hline
\end{tabular}

Table 5. Proposed Precision for Fruits Image

\begin{tabular}{|l|l|l|l|l|l|}
\hline \multirow{2}{*}{$\begin{array}{l}\text { Similarity } \\
\text { Measure }\end{array}$} & \multicolumn{5}{|l|}{ Proposed Precision (\%) } \\
\cline { 2 - 6 } & $\mathbf{1 0}$ & $\mathbf{2 0}$ & $\mathbf{3 0}$ & $\mathbf{4 0}$ & $\mathbf{5 0}$ \\
\hline ED & 81.31 & 85.11 & 81.20 & 87.36 & 81.31 \\
\hline CBD & 75.41 & 79.17 & 80.73 & 76.52 & 79.44 \\
\hline
\end{tabular}




\begin{tabular}{|l|l|l|l|l|l|}
\hline CD & 81.82 & 84.00 & 78.45 & 77.52 & 79.63 \\
\hline MD & 77.98 & 77.06 & 83.49 & 81.73 & 74.80 \\
\hline
\end{tabular}

Table 6. Base Precision for Fruits Image

\begin{tabular}{|l|l|l|l|l|l|}
\hline Similarity & \multicolumn{5}{|l|}{ Base Precision (\%) } \\
\cline { 2 - 6 } Measure & $\mathbf{1 0}$ & $\mathbf{2 0}$ & $\mathbf{3 0}$ & $\mathbf{4 0}$ & $\mathbf{5 0}$ \\
\hline ED & 83.45 & 81.17 & 82.96 & 82.52 & 81.82 \\
\hline
\end{tabular}

Table 7. Proposed Precision for Daintree Image

\begin{tabular}{|l|l|l|l|l|l|}
\hline \multirow{2}{*}{$\begin{array}{l}\text { Similarity } \\
\text { Measure }\end{array}$} & \multicolumn{5}{|l|}{ Proposed Precision (\%) } \\
\cline { 2 - 6 } & $\mathbf{1 0}$ & $\mathbf{2 0}$ & $\mathbf{3 0}$ & $\mathbf{4 0}$ & $\mathbf{5 0}$ \\
\hline ED & 79.46 & 79.82 & 78.57 & 77.88 & 86.27 \\
\hline CBD & 77.88 & 85.26 & 78.26 & 80.61 & 80.65 \\
\hline CD & 76.15 & 83.33 & 77.48 & 85.00 & 85.00 \\
\hline MD & 76.42 & 78.05 & 78.81 & 88.07 & 76.72 \\
\hline
\end{tabular}

Table 8. Precision and Accuracy is Compared using Base[11] and Proposed System

\begin{tabular}{|l|l|l|l|l|}
\hline Category & $\begin{array}{l}\text { Base } \\
\text { Precision(\%) }\end{array}$ & $\begin{array}{l}\text { Proposed } \\
\text { Precision(\%) }\end{array}$ & $\begin{array}{l}\text { Base } \\
\text { Accuracy } \\
(\%)\end{array}$ & $\begin{array}{l}\text { Proposed } \\
\text { Accuracy } \\
(\%)\end{array}$ \\
\hline $\begin{array}{l}\text { Sports } \\
\text { 218.jpg }\end{array}$ & 81.95 & 83.67 & 75.55 & 77.05 \\
\hline $\begin{array}{l}\text { Flower } \\
\text { 278.jpg }\end{array}$ & 79.72 & 84.21 & 77.15 & 79.74 \\
\hline $\begin{array}{l}\text { Fruits } \\
\text { 725.jpg }\end{array}$ & 81.16 & 84.82 & 75.35 & 78.96 \\
\hline $\begin{array}{l}\text { Tools } \\
\text { 750.jpg }\end{array}$ & 81.95 & 86.73 & 76.95 & 79.07 \\
\hline
\end{tabular}

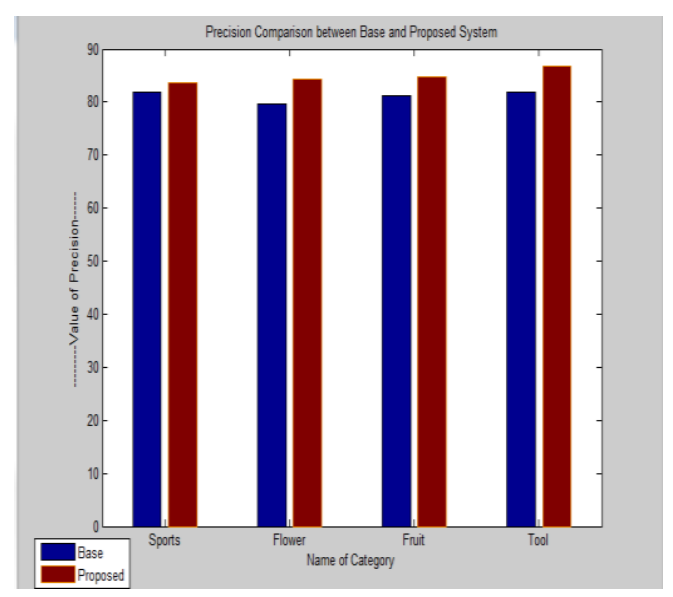

Figure 6. Comparison between Base[11] and Proposed System

\section{Conclusion}

On this paper, we offered a wavelet-situated salient point extraction algorithm and utilized it in the CBIR. The salient features are intriguing for picture retrieval on account that they're placed in visible focal point facets and accordingly they can capture the neighborhood picture information. In this research, an efficient low level features of 
CBIR using BCI with color coding (CC), GLDM, Hu moments and four levels of DWT. Experimental outcomes present that the proposed method increases the accuracy and image retrieval precision. The work can be comprehensive further to develop the proposed process by considering partial overlapping region with the ROI and using more effective feature set to symbolize dissimilar regions in the image. We approved that the retrieval supported the new invariants considerably outmatch the extra a set of ancient ways included in our tests.

\section{References}

[1] F. Long, H. Zhang, and D. D. Feng, "Fundamentals of Content-BasedImage Retrieval," Multimedia Information Retrieval and Management, Springer, Berlin, 2003.

[2] Prashant Srivastava, Om Prakash, and Ashish Khare "Content-Based Image Retrieval Using Moments of WaveletTransform" The 2014 International conference on control, Automation and Information Sciences (ICCAIS 2014) December 2-5 Gwangju South Korea.

[3] P. S. Suhasini, K. S. R. Krishna, and I. V. M. Krishna, "CBIR UsingColor Histogram Processing," Journal of Theoretical and AppliedInformation Technology, vol. 16, no. 1, pp. 116-122, 2009.

[4] J. Huang, S. R. Kumar, M. Mitra, and Wei-Jing Zhu, "Image Indexingusing Color Correlograms," U.S. Patent 6,246,790, June 12, 2001.

[5] S. Manjunath and W. X. Ma, "Browsing and Retrieval of ImageData," IEEE Trans. On Pattern Analysis and Machine Intelligence,vol. 18, no. 8, pp. 837-842, August 1996.

[6] Zhang and G. Lu, "Generic, Fourier Descriptor for Shape-based ImageRetrieval. Signal ProcessingImage Communication," vol. 17, no. 10,November 2002.

[7] M. Khare, R. K. Srivastava, and A. Khare, "Moving ObjectSegmentation In Daubechies Complex Wavelet Domain. Signal,Image and Video Processing," Accepted, Springer, 2013.

[8] Deepti Yadav*, Chavi Singhal "Offline Signature Recognition Using PCA-NN Method and GLDM Feature Extraction" International Journal of Advanced Research in Computer Science and Software EngineeringVolume 5, Issue 9, September 2015.

[9] Nilesh Y. Choudhary,Mrs. Rupal Patil, Dr. Umesh. Bhadade, Prof. Bhupendra M Chaudhari, ${ }^{\text {ece }}$ Signature Recognition \& Verification System Using Back Propagation Neural Network ${ }^{\text {eee }}$ Volume 2, No. 1, January 2013,IJIEASR.

[10] Jeyanthi Prabhu and Jawahar Senthil Kumar "Wavelet Based Content Based Image Retrieval Using Color And Texture Feature Extraction By Gray Level Coocurence Matrix And Color Coocurence Matrix" Journal of Computer Science 10 (1): 15-22, 2014.

[11] Kommineni Jenni, Satria Mandala, Mohd Shahrizal Sunar "Content Based Image Retrieval Using Colour Strings Comparison" Content Based Image Retrieval Using Colour Strings Comparison.

[12] Ekta Gupta, Rajendra Singh Kushwah "Combination of Global and Local Features usingDWT with SVM for CBIR" 2015 IEEE.

[13] Abdulqader Mahmoud, Hamza Al Maharmeh and Hussain Al-Ahmad "A New Watermarking Algorithm for Scanned Colored PDF Files Using DWT and Hash Function" 2015 International Conference on Information and Communication Technology Research (ICTRC2015).

[14] Kommineni Jenni, Satria Mandala, Mohd Shahrizal Sunar "Content Based Image Retrieval Using Colour Strings Comparison"Content Based Image Retrieval Using Colour Strings Comparison.

[15] M. Lamard, G. Cazuguel, G. Quellec, L. Bekri, C. Roux, and B.Cochener, "Content based Image Retrieval Based on WaveletTransform Coefficients Distribution," 29th Annual InternationalConference on Engineering in Medicine and Biology Society, IEEE,pp. 4532-4535.

[16] L. Flores-Pulido, O. Starostenko, D. Flores-Quéchol, J. I. Rodrigues-Flores, Ingrid Kirschning, and J. A. Chávez-Aragón, "Content-basedImage Retrieval Using Wavelets," Proceedings of the 2nd WSEASInternational Conference on Computer Engineering and Applications,pp. 40-45, 2008. 